MONOCLONAL GAMMOPATHY OF UNDETERMINED SIGNIFICANCE AND BONE HEALTH OUTCOMES: A SYSTEMATIC REVIEW AND

\title{
EXPLORATORY META-ANALYSIS
}

Running head: MGUS and fractures

Nicola Veronese ${ }^{1}$, Claudio Luchini ${ }^{2}$, Marco Solmi ${ }^{3}$, Giuseppe Sergi ${ }^{1}$, Enzo Manzato ${ }^{1}$, Brendon Stubbs $^{4-6}$

${ }^{1}$ Department of Medicine, Geriatrics Section, University of Padova, Italy.

${ }^{2}$ Department of Pathology and Diagnostics, University and Hospital Trust of Verona, Verona, Italy.

${ }^{3}$ Department of Neurosciences, University of Padova, Padova, Italy.

${ }^{4}$ Physiotherapy Department, South London and Maudsley NHS Foundation Trust, Denmark Hill, London SE5 8AZ, United Kingdom.

${ }^{5}$ Health Service and Population Research Department, Institute of Psychiatry, King's College London, De Crespigny Park, Box SE5 8 AF, London, United Kingdom.

${ }^{6}$ Faculty of Health, Social Care and Education, Anglia Ruskin University, Chelmsford, UK.

\section{Correspondence to:}

Nicola Veronese, University of Padova, Department of Medicine, Geriatrics Section, Via Giustiniani, 235128 Padova, Italy. Email: ilmannato@gmail.com 


\begin{abstract}
Monoclonal gammopathy of undetermined significance (MGUS) is a common condition in the elderly. A number of studies have investigated the relationship between MGUS and bone health outcomes including bone mineral density (BMD), osteoporosis and fractures, but no meta-analysis exists. We conducted a systematic review and exploratory meta-analysis comparing bone health outcomes in patients with MGUS.

Two independent authors searched PubMed and Scopus from inception until October 19, 2016. A meta-analysis of cross-sectional and longitudinal studies investigating fractures and BMD was conducted. Standardized mean differences (SMD) $\pm 95 \%$ and confidence intervals (CIs) were calculated for BMD, and risk ratios (RRs) were calculated for prevalent and incident fractures. Of 174 initial hits, 10 studies of moderate methodological quality were eligible, including 8,711 individuals with MGUS vs. 52,865 controls. Compared to controls, subjects with MGUS showed significantly lower values for radial cortical volumetric BMD (1 study; SMD=-5.45, 95\%CI: -7.24 to -3.66), but not at the lumbar spine, femoral neck or hip. The incidence of fractures was higher in people with MGUS ( $n=7,466)$ versus controls $(n=52,304)\left(R R=1.36,95 \%\right.$ CI: $\left.1.28-1.44, I^{2}=0 \%\right)$ over a median of 12.5 year follow up. The incidence of vertebral fractures was particularly elevated (RR=2.50, 95\%CI: 1.53-4.06) although limited to two studies.

In conclusion, although with limitations, our preliminary meta-analysis suggests that patients with MGUS are at higher risk of fractures despite evidence for differences in BMD being equivocal. Future longitudinal research is required to confirm our findings and determine if fracture prevention interventions are warranted in people with MGUS.
\end{abstract}

Keywords: Monoclonal gammopathy of undetermined significance; osteoporosis; fractures; aging. 


\section{INTRODUCTION}

Monoclonal gammopathy of undetermined significance (MGUS) (defined by a serum monoclonal protein level of $\leq 3.0 \mathrm{~g} / \mathrm{dL},<10 \%$ bone marrow plasma cells, and the absence of plasma cell proliferation-related complications) is a common condition[1], especially in the elderly in which reaches a prevalence of about $8 \%$ in those older than 85 years.[2] Approximately 1\% of people affected by MGUS progress to a diagnosis of multiple myeloma (MM) every year.[2]

Whilst the majority of research to date has considered the progression from MGUS to MM, recent research has also demonstrated that MGUS appears to be associated with bone health outcomes, and in particular osteoporosis and fractures.[3] Reasons for this relationship include the fact that MGUS seems to be associated with an uncoupling in osteoclasts (increased) and osteoblasts (decreased) balance and a higher production of pro-inflammatory and bone reabsorptive cytokines thus increasing the probability of osteoporosis and fractures.[3, 4]

However, the literature considering MGUS and bone health outcomes is equivocal and no quantitative synthesis exists. Therefore, we conducted a systematic review and exploratory meta-analysis investigating the association between MGUS and osteoporosis, bone mineral density (BMD) and fractures risk. We hypothesized that people with MGUS are at increased risk of poor bone health outcomes.

\section{METHODS}

This systematic review was conducted following the Preferred Reporting Items for Systematic reviews and Meta-Analyses (PRISMA) statement.[5]

\section{Search Strategy}

Two investigators (NV and CL) independently and blinded of the other investigator's decision searched PubMed and Scopus, without language restriction, from database inception until 10/19/16, for studies comparing the presence of fractures and BMD values between patients with MGUS vs. 
controls. Any inconsistencies were resolved by consensus with a third author (BS) available for mediation. In PubMed, we used the following search strategy: ("Monoclonal Gammopathy” OR “MGUS”) AND ("bone mineral density" OR "bone density” OR "BMD” OR "fracture*" OR "osteoporosis" OR “dxa” OR “Absorptiometry, X-Ray”). In Scopus the search used was: “TITLEABS-KEY ( "Monoclonal Gammopathy" OR "MGUS" ) AND TITLE-ABS-KEY ( "bone mineral density" OR "bone density" OR "BMD" OR "fracture*" OR "osteoporosis" OR "dxa" OR "Absorptiometry, X-Ray" )”. Reference lists of included articles and those relevant to the topic were hand-searched for identification of additional, potentially relevant articles.

\section{Study Selection}

We included cross-sectional and/ or longitudinal studies comparing data on osteoporosis, fractures, BMD, and T-scores (deviation from the bone mass of an average healthy 30 year old adult)[6] between patients with MGUS vs. controls. Data about bone outcomes in patients with MGUS were summarized as prevalence. In case of doubled study, the most recent was selected. Specific inclusion criteria were: (1) reported BMD values or fractures, (2) validated diagnosis of MGUS and (3) published in a peer-review journal. Studies were excluded if they (1) measured only in vitro parameters (e.g. bone cellular markers) or used animal models or (2) reported only laboratory data (i.e. serum laboratory markers of bone deposition or reabsorption). The corresponding author was contacted at least two times in a month for additional information, if needed.

\section{Data Extraction}

Two authors (GS, EM) independently extracted data using a standardized spreadsheet. Any disagreement was resolved by consensus. The following information were extracted: i) study population characteristics, ii) duration of follow-up (for longitudinal studies), iii) clinical setting in which the study was performed, iv) percentage of IgG monoclonal band, v) main characteristics of 
MGUS and control groups (age, percentage of women and body mass index), vi) matching variables used, and vi) quality scores.

\section{Quality assessment}

The Newcastle Ottawa Scale (NOS) was used to assess the quality of included studies. [7] However the item "demonstration that outcome of interest was not present at start of study" was omitted as it is not appropriate.

\section{Statistical Analysis}

The primary outcomes were the prevalence or incidence of fractures and characteristics of BMD parameters, comparing those with MGUS vs. controls. When combining studies, the random effects model was used to account for study heterogeneity.[8] For BMD data, standardized mean difference (SMD) with its 95\% confidence interval (CI) was used, as well as risk ratios (RRs) for prevalent or incident fractures.

Study heterogeneity was measured using the chi-squared and I-squared statistics, with chi-squared $\mathrm{p}<0.05$ and I-squared $\geq 50 \%$ indicating the presence of significant heterogeneity.[9] We anticipated conducting meta-regression analysis for outcomes including $\geq 4$ studies. The meta-analysis was performed using Comprehensive Meta-Analysis V3.

\section{RESULTS}

The search identified 184 non duplicate potentially eligible studies. After excluding 164 papers through title and abstract review and 10 from full text examination, 10 studies[10-19] were included (Supplementary Figure 1). 


\section{Study and Patient Characteristics}

Studies and patient characteristics are summarized in Supplementary Table 1 (cross-sectional) and 2 (longitudinal). The 9 analyzed studies included a total of 8,711 participants with MGUS (mainly with an IgG component) vs. 52,865 controls. The mean age of those with MGUS was 70 (range 61.373.5) and 54\% females.

All of the studies comparing MGUS vs. control group were matched for age and and/ or sex. The majority of the studies were made in Europe or in North America. Cross-sectional surveys were predominantly conducted among outpatients, while longitudinal included only community-dwellers.

\section{Methodological quality of included studies}

The quality of the studies was good, since among 8 points possible available, the median for crosssectional and longitudinal studies was 5 and 7, respectively (Supplementary Tables 1-3).

\section{Cross-sectional bone health outcomes}

Four studies [12-15] reported an overall prevalence of fractures of 14\% (95\%CI: 3-52) in MGUS participants, while the prevalence of osteoporosis in 3 studies[12, 13, 19] was 14\% (95\%CI:2-62) (Supplementary Figures 1-2).

As reported in Table 1, BMD (lumbar spine, hip and femoral neck) and T-scores (lumbar spine and hip) were not different between MGUS and controls. Regarding pQCT findings, one study[11] reported that MGUS patients had significantly lower radial cortical volumetric BMD than controls (SMD=-5.45, 95\%CI: -7.24 to -3.66). Data from another study[12] demonstrated that MGUS patients had a significantly higher prevalence of total $(\mathrm{RR}=2.51,95 \% \mathrm{CI}$ : $1.89-3.33)$ and vertebral fractures (RR=2.30, 95\%CI: 1.71-3.11) (Table 2). 


\section{Longitudinal studies and incidence of fractures}

Over a median follow up period of 12.5 years, data were pooled across three studies [16-18] among 7,466 people with MGUS and 52,304 controls, demonstrating a significant higher incidence of fractures versus controls ( $\mathrm{RR}=1.36,95 \% \mathrm{CI}$ : $\left.1.28-1.44, \mathrm{I}^{2}=0 \%\right)$. The risk of vertebral fractures was particularly elevated among 5,931 people with MGUS and 36,594 controls (2 studies[16, 17]; $\left.\mathrm{RR}=2.50,95 \% \mathrm{CI}: 1.53-4.06, \mathrm{I}^{2}=80 \%\right)$.

\section{DISCUSSION}

To the best of our knowledge this is the first systematic review and meta-analysis investigating the relationship between MGUS and bone health outcomes.

Despite the limited number of studies included, our data suggests that people with MGUS have a higher prevalence and incidence of fractures (particularly vertebral) and lower cortical BMD, while the BMD (assessed with DXA) seems not to be affected by this pathological condition. A possible explanation of this finding is that patients with MGUS have some effect of degenerative changes (particularly vertebral wedging/compressions) that are well-known pitfalls in the correct interpretation of DXA results. Another possible explanation is that DXA is not able to measure microarchitectural changes (like cortical porosity) at central sites as observed in the HRpQCT studies at peripheral site (like radius). Therefore, our findings suggest that the BMD measurements with DXA might not be reliable in people with MGUS.

Over a median follow-up of 12.5 years including almost 60,000 people, we found that patients with MGUS are at 36\% and over double the risk of any and vertebral fractures. The reasons for this increased risk of fractures among people with MGUS are yet to be elucidated. However, one possible reason is that among patients with MGUS, the reduction in cortical BMD observed is not counterbalanced by an increase in trabecular component, thus providing only a partial biomechanical adaptation to limit the overall loss of bone strength.[20] Another potential hypothesis for the increased fracture rate among people with MGUS is that a significant percentage develop a multiple myeloma 
during follow-up period and it is well-known that patients with this condition have a high presence of pathological fractures.[21] It seems that patients with MGUS and multiple myeloma share some common pathways that could increase osteoclasts [3] [e.g. receptor activator for NF-kB ligand (RANKL), chemokine (C-C) ligand motif 3 (CCL3)/macrophage inflammatory protein (MIP)-1a] and decreasing osteoblasts activity [3] [e.g. Wnt signalling antagonists Dickkopf-related protein 1 (DKK1), soluble frizzled related proteins 2 and 3, and sclerostin]. Whilst this might possibly explain our results, the clinical impact of these alterations seem to be contradictory, since Drake observed that some studies reported significant differences in bone turnover in MGUS compared to controls, while another study did not.[3] Therefore, clearly future research is needed to disentangle these relationships and confirm the increased incidence of fractures in MGUS patients, whilst also attempting to disentangle potential reasons for this.

The findings of our meta-analysis should be interpreted within its clear limitations. The main is the small number of studies within each outcome. This not only means that our results should be considered preliminary, but this also precluded us from investigating publication bias. Of particular importance is that publication bias is a very common problem due to the presence of studies reporting significant positive associations [22], that we unable to address due to the limited number of the studies available for each outcome. Thus, we think that this factor could play a role in the interpretation of our results Second, within the longitudinal studies, it remains unclear how many people with MGUS developed multiple myeloma, which could be of importance to differentiate osteoporotic and pathological fractures. Third, the assessment of potential confounders (like age, medications and renal function) is limited to a single study and might influence fracture rates in people with MGUS. Finally, although we identified a high heterogeneity for several outcomes, we were not able to run any meta-regression analysis for identifying potential moderators due to the limited number of studies for each outcome. Among the strengths of our work we could consider the fact that it is the first meta-analysis assessing the impact of MGUS on bone health outcomes and the large number of the participants included. 
In conclusion, from our preliminary meta-analysis, it appears that patients with MGUS are at higher risk of future fractures despite evidence for differences in BMD being less apparent. Future research is required to build on our results. In clinical practice, people with MGUS might benefit from interventions to prevent osteoporosis and fractures. 
Acknowledgments.

Funding source: none.

Financial Disclosure: none.

Conflict of Interest: All authors have no conflict of interest. 


\section{REFERENCES}

1. Kyle RA, Durie BGM, Rajkumar S V, et al (2010) Monoclonal gammopathy of undetermined significance (MGUS) and smoldering (asymptomatic) multiple myeloma: IMWG consensus perspectives risk factors for progression and guidelines for monitoring and management. Leukemia 24:1121-7. doi: 10.1038/leu.2010.60

2. Kyle RA, Therneau TM, Rajkumar SV, et al (2006) Prevalence of monoclonal gammopathy of undetermined significance. The New England journal of medicine 354:1362-9. doi: 10.1056/NEJMoa054494

3. Drake MT (2014) unveiling skeletal fragility in patients diagnosed with MGUS: no longer a condition of undetermined significance? Journal of bone and mineral research : the official journal of the American Society for Bone and Mineral Research 29:2529-33. doi: 10.1002/jbmr.2387

4. Drake MT (2009) Bone disease in multiple myeloma. Oncology (Williston Park, NY) 23:2832.

5. Liberati A, Altman DG, Tetzlaff J, et al (2009) The PRISMA statement for reporting systematic reviews and meta-analyses of studies that evaluate health care interventions: explanation and elaboration. PLoS medicine 6:e1000100. doi: 10.1371/journal.pmed.1000100

6. Organization WH (2004) WHO SCIENTIFIC GROUP ON THE ASSESSMENT OF OSTEOPOROSIS AT PRIMARY HEALTH Care Level. World Health Organization May:57. doi: 10.1016/S0140-6736(02)08761-5

7. Wells G, Shea B, O’Connell D, et al (2012) The Newcastle-Ottawa Scale (NOS) for assessing the quality if nonrandomized studies in meta-analyses. (Available from: URL: http://www.ohri.ca/programs/clinical_epidemiology/oxford.asp) 2012. doi: 10.2307/632432

8. DerSimonian R, Laird N (1986) Meta-analysis in clinical trials. Controlled clinical trials 7:177-188. doi: 10.1016/0197-2456(86)90046-2 
9. Higgins JPT, Thompson SG (2002) Quantifying heterogeneity in a meta-analysis. Statistics in Medicine 21:1539-1558. doi: 10.1002/sim.1186

10. Dizdar O, Erman M, Cankurtaran M, et al (2008) Lower bone mineral density in geriatric patients with monoclonal gammopathy of undetermined significance. Annals of hematology 87:57-60. doi: 10.1007/s00277-007-0376-5

11. Ng AC, Khosla S, Charatcharoenwitthaya N, et al (2011) Bone microstructural changes revealed by high-resolution peripheral quantitative computed tomography imaging and elevated DKK1 and MIP-1 $\alpha$ levels in patients with MGUS. Blood 118:6529-34. doi: 10.1182/blood-2011-04-351437

12. Pepe J, Petrucci MT, Nofroni I, et al (2006) Lumbar bone mineral density as the major factor determining increased prevalence of vertebral fractures in monoclonal gammopathy of undetermined significance. British journal of haematology 134:485-90. doi: 10.1111/j.13652141.2006.06217.x

13. Abrahamsen B, Andersen I, Christensen SS, et al (2005) Utility of testing for monoclonal bands in serum of patients with suspected osteoporosis: retrospective, cross sectional study. BMJ (Clinical research ed) 330:818. doi: 10.1136/bmj.38376.401701.8F

14. Golombick T, Diamond T (2008) Prevalence of monoclonal gammopathy of undetermined significance/myeloma in patients with acute osteoporotic vertebral fractures. Acta haematologica 120:87-90. doi: 10.1159/000162282

15. Piot JM, Royer M, Schmidt-Tanguy A, et al (2015) Factors associated with an increased risk of vertebral fracture in monoclonal gammopathies of undetermined significance. Blood cancer journal 5:e345. doi: 10.1038/bcj.2015.71

16. Bida JP, Kyle RA, Therneau TM, et al (2009) Disease associations with monoclonal gammopathy of undetermined significance: a population-based study of 17,398 patients. Mayo Clinic proceedings 84:685-93. doi: 10.1016/S0025-6196(11)60518-1

17. Kristinsson SY, Tang M, Pfeiffer RM, et al (2010) Monoclonal gammopathy of 
undetermined significance and risk of skeletal fractures: a population-based study. Blood 116:2651-5. doi: 10.1182/blood-2010-04-282848

18. Gregersen H, Jensen P, Gislum M, et al (2006) Fracture risk in patients with monoclonal gammopathy of undetermined significance. British journal of haematology 135:62-7. doi: 10.1111/j.1365-2141.2006.06269.x

19. Malgo F, Appelman-Dijkstra NM, Termaat MF, et al (2016) High prevalence of secondary factors for bone fragility in patients with a recent fracture independently of BMD. Archives of Osteoporosis 11:1-8. doi: 10.1007/s11657-016-0258-3

20. Seeman E, Delmas PD (2006) Bone quality--the material and structural basis of bone strength and fragility. The New England journal of medicine 354:2250-61. doi: 10.1056/NEJMra053077

21. Walker RE, Lawson MA, Buckle CH, et al (2014) Myeloma bone disease: pathogenesis, current treatments and future targets. British medical bulletin 111:117-38. doi: 10.1093/bmb/ldu016

22. Dalton JE, Bolen SD, Mascha EJ (2016) Publication Bias: The Elephant in the Review. Anesthesia \& Analgesia 123: 